\title{
CALDERÓN-ZYGMUND OPERATORS WITH KERNELS OF DINI'S TYPE AND THEIR MULTILINEAR COMMUTATORS ON GENERALIZED VARIABLE EXPONENT MORREY SPACES
}

\author{
VAGIF S. GULIYEV AND AFAG F. ISMAYILOVA
}

\begin{abstract}
Let $T$ be a Calderón-Zygmund operator of type $\omega$ with $\omega(t)$ being nondecreasing and satisfying a kind of Dini's type condition and let $T_{\vec{b}}$ be the multilinear commutators of $T$ with $B M O^{m}$ functions. In this paper, we study the boundedness of the operators $T$ and $T_{\vec{b}}$ on generalized variable exponent Morrey spaces $M^{p(\cdot), \varphi}$. We find the sufficient conditions on the pair $\left(\varphi_{1}, \varphi_{2}\right)$ with $\vec{b} \in B M O^{m}\left(\mathbb{R}^{n}\right)$ which ensures the boundedness of the operators $T$ and $T_{\vec{b}}$ from $M^{p(\cdot), \varphi_{1}}$ to $M^{p(\cdot), \varphi_{2}}$.
\end{abstract}

\section{Introduction}

The theory of Calderón-Zygmund operators has played very important roles in modern harmonic analysis with lots of extensive applications in the others fields of mathematics, which has been extensively studied (see [3, 4, 6, 29, 30, 39]). In particular, Yabuta introduced certain $\omega$-type Calderón-Zygmund operators to facilitate his study of certain classes of pseudodifferential operators (see [43]). Let $\omega$ be a non-negative and non-decreasing function on $\mathbb{R}_{+}=(0, \infty)$. We say that $\omega$ satisfies the Dini condition and write $\omega \in D i n i$, if

$$
\int_{0}^{\infty} \frac{\omega(t)}{t} d t<\infty
$$

A measurable function $K(\cdot, \cdot)$ on $\mathbb{R}^{n} \times \mathbb{R}^{n}$ is said to be a $\omega$-type Calderón-Zygmund kernel if it satisfies

$$
|K(x, y)| \leq C|x-y|^{-n}
$$

and for all distinct $x, y \in \mathbb{R}^{n}$, and all $z$ with $2|x-z|<|x-y|$, there exist positive constants $C$ and $\gamma$ such that

$$
|K(x, y)-K(z, y)|+|K(y, x)-K(y, z)| \leq C \omega\left(\frac{|x-z|}{|x-y|}\right)|x-y|^{-n} .
$$

Definition 1.1. Let $T$ be a linear operator from $\mathcal{S}\left(\mathbb{R}^{n}\right)$ into its dual $\mathcal{S}^{\prime}\left(\mathbb{R}^{n}\right)$, where $\mathcal{S}\left(\mathbb{R}^{n}\right)$ denotes the Schwartz class. One can say that $T$ is a $\omega$-type CalderónZygmund operator if it satisfies the following conditions:

2010 Mathematics Subject Classification. 42B20, $42 \mathrm{~B} 35$.

Key words and phrases. Generalized variable exponent Morrey spaces; Calderón-Zygmund operator; commutator; BMO. 
i) $T$ can be extended to be a bounded linear operator on $L_{2}\left(\mathbb{R}^{n}\right)$;

ii) there is a $\omega$-type Calderón-Zygmund kernel $K(\cdot, \cdot)$ such that

$$
T f(x)=\int_{\mathbb{R}^{n}} K(x, y) f(y) d y, \text { as } f \in C_{c}^{\infty} \text { and } x \notin \operatorname{supp} f .
$$

It is easy to see that the classical Calderón-Zygmund operator with standard kernel is a special case of $\omega$-type operator $T$ as $\omega(t)=t^{\varepsilon}$ with $0<\varepsilon \leq 1$. Given a locally integrable function $b$, the commutator generated by $T$ and $b$ is defined by

$$
[b, T] f(x):=b(x) T f(x)-T(b f)(x)=\int_{\mathbb{R}^{n}}(b(x)-b(y)) K(x, y) f(y) d y .
$$

Let $\vec{b}=\left(b_{1}, \ldots, b_{m}\right)$ and $b_{j}, 1 \leq j \leq m$ be locally integrable functions when we consider multilinear commutators as defined by

$$
T_{\vec{b}} f(x)=\int_{\mathbb{R}^{n}} \prod_{j=1}^{m}\left(b_{j}(x)-b_{j}(y)\right) K(x, y) f(y) d y .
$$

Furthermore, if we take $b_{i}=b, i=1, \ldots, m$, then we define the following integral equation

$$
T_{\vec{b}} f(x)=\int_{\mathbb{R}^{n}}(b(x)-b(y))^{m} K(x, y) f(y) d y=[b, T]^{m} f(x) .
$$

Integral operators of Calderón-Zygmund kind appear in the representation formulas of the solutions of various PDEs. Thus the continuity of the CalderónZygmund integral in certain functional space permit to study the regularity of the solutions of boundary value problems for linear PDEs in the corresponding space, see, for example, $[5,14,12,35]$.

The classical Morrey spaces were introduced by Morrey [35] to study the local behavior of solutions to second-order elliptic partial differential equations. Moreover, various Morrey spaces are defined in the process of study. The first author, Mizuhara and Nakai $[15,33,36]$ introduced generalized Morrey spaces $M^{p, \varphi}\left(\mathbb{R}^{n}\right)$ (see, also $[16,38])$.

Variable exponent function spaces (see [8]) received considerable attentions in recent decades. They are important not only in theory as generalizations of classical function spaces, but also for their wide applications in the fields of fluid dynamics, elasticity dynamics, the differential equations with nonstandard growth. The rich development can be found in many research works of the theory of variable exponent function spaces. We refer to $[2,8,10,23,26,42]$ for the details. For example, Lebesgue spaces with variable exponent were studied in $[7,9,24,27,40]$, Morrey spaces with variable exponent were studied in [2, 13, 34] and generalized Morrey spaces with variable exponent were studied in $[1,11,19$, $20,21]$.

The main purpose of this paper is to establish a number of results concerning variable exponent Morrey boundedness of Calderón-Zygmund operators with kernels of mild regularity. Let $T$ be a linear Calderón-Zygmund operator of type $\omega(t)$ with $\omega$ being nondecreasing and $\omega \in$ Dini, but without assuming to be concave. We show that the $\omega$-type Calderón-Zygmund operators $T$ and their multilinear commutators $T_{\vec{b}}$ are bounded from one generalized variable exponent 
Morrey space $M^{p(\cdot), \varphi_{1}}$ to another $M^{p(\cdot), \varphi_{2}}$. We find the sufficient conditions on the pair $\left(\varphi_{1}, \varphi_{2}\right)$ with $\vec{b} \in B M O^{m}\left(\mathbb{R}^{n}\right)$ which ensures the boundedness of the operators $T$ and $T_{\vec{b}}$ from $M^{p(\cdot), \varphi_{1}}$ to $M^{p(\cdot), \varphi_{2}}$.

By $A \lesssim B$ we mean that $A \leq C B$ with some positive constant $C$ independent of appropriate quantities. If $A \lesssim B$ and $B \lesssim A$, we write $A \approx B$ and say that $A$ and $B$ are equivalent.

\section{Some definitions and auxiliary results}

Let $\mathbb{R}^{n}$ be the $n$-dimensional Euclidean space of points $x=\left(x_{1}, \ldots, x_{n}\right)$ with norm $|x|=\left(\sum_{i=1}^{n} x_{i}^{2}\right)^{1 / 2}$. For $x \in \mathbb{R}^{n}$ and $r>0$, denote $B(x, r)$ the open ball centered at $x$ of radius $r$. Let ${ }^{\complement} B(x, r)$ be the complement of the ball $B(x, r)$, and $|B(x, r)|$ be the Lebesgue measure of $B(x, r)$. For a measurable set $E$, we define the Lebesgue measure of $E$ by $|E|$, and the characteristic function of $E$ by $\chi_{E}$.

Given an open set $E \subset \mathbb{R}^{n}$, and a measurable function $p(\cdot): E \rightarrow[1, \infty), p^{\prime}(\cdot)$ is the conjugate exponent defined by $p^{\prime}(\cdot)=p(\cdot) /(p(\cdot)-1)$. For a measurable subset $E \subset \mathbb{R}^{n}$, we denote $p^{-}(E)=\operatorname{ess} \inf \{p(x): x \in E\}, p^{+}(E)=\operatorname{ess} \sup \{p(x)$ : $x \in E\}$. Especially, we denote $p^{-}=p^{-}\left(\mathbb{R}^{n}\right)$ and $p^{+}=p^{+}\left(\mathbb{R}^{n}\right)$. The set $\mathcal{P}(E)$ consists of all $p(\cdot): E \rightarrow[1, \infty)$ satisfying $p^{-}(E)>1, p^{+}(E)<\infty$. Similarly we denote by $\mathcal{P}\left(\mathbb{R}^{n}\right)$ the set of all measurable functions $p(\cdot): \mathbb{R}^{n} \rightarrow(1, \infty)$ such that $1<p^{-} \leq p(x) \leq p^{+}<\infty$. Denote by $\mathcal{P}_{0}\left(\mathbb{R}^{n}\right)$ the set of all measurable functions $p(\cdot): \mathbb{R}^{n} \rightarrow(0, \infty)$ such that $0<p^{-} \leq p(x) \leq p^{+}<\infty, x \in \mathbb{R}^{n}$. Let $\mathcal{P}_{1}\left(\mathbb{R}^{n}\right)$ be the set of all measurable functions $p(\cdot): \mathbb{R}^{n} \rightarrow[1, \infty)$ such that $1 \leq p^{-} \leq p(x) \leq p^{+}<\infty$.

We define the variable exponent Lebesgue space $L^{p(\cdot)}(E)$ as the set of realvalued measurable functions $f$ on $E$ such that, for some $\varepsilon>0, \int_{E}(\varepsilon|f(x)|)^{p(x)} d x<$ $\infty$. This is a Banach function space with respect to the Luxemburg-Nakano norm,

$$
\|f\|_{L^{p(\cdot)}(E)}=\inf \left\{\lambda>0: \int_{E}\left(\frac{f(x) \mid}{\lambda}\right)^{p(x)} d x \leq 1\right\} .
$$

The space $L_{\text {loc }}^{p(\cdot)}(E)$ is defined by

$L_{\text {loc }}^{p(\cdot)}(\Omega):=\left\{f\right.$ is measurable $: f \in L^{p(\cdot)}(E)$ for all compact subsets $\left.E \subset \Omega\right\}$.

Next we define some classes of variable exponent functions. The set $\mathcal{B}\left(\mathbb{R}^{n}\right)$ consists of all measurable functions $p(\cdot) \in \mathcal{P}\left(\mathbb{R}^{n}\right)$ satisfying that the HardyLittlewood maximal operator $M$ is bounded on $L^{p(\cdot)}\left(\mathbb{R}^{n}\right)$.

An important subset of $\mathcal{B}\left(\mathbb{R}^{n}\right)$ is the class of globally log-Hölder continuous functions $p(\cdot) \in L H\left(\mathbb{R}^{n}\right)$, with $p(\cdot) \in \mathcal{P}\left(\mathbb{R}^{n}\right)$. Recall that $p(\cdot) \in L H\left(\mathbb{R}^{n}\right)$, if $p(\cdot)$ satisfies

$$
\begin{aligned}
& |p(x)-p(y)| \leq \frac{C}{-\log (|x-y|)},|x-y| \leq 1 / 2 \text { and } \\
& |p(x)-p(y)| \leq \frac{C}{\log (e+|x|)},|y| \geq|x| .
\end{aligned}
$$

We will also make use of the estimate provided by the following inequality (see [20, Lemma 2.2], [21, Theorem 3.2]). 


$$
\left\|\chi_{B(x, r)}(\cdot)\right\|_{L^{p(\cdot)\left(\mathbb{R}^{n}\right)}} \leq C r^{\theta_{p}(x, r)}, \quad x \in \mathbb{R}^{n}, p \in L H\left(\mathbb{R}^{n}\right) \cap \mathcal{P}\left(\mathbb{R}^{n}\right),
$$

where $\theta_{p}(x, r)=\left\{\begin{array}{l}\frac{n}{p(x)}, r \leq 1, \\ \frac{n}{p(\infty)}, r \geq 1\end{array}\right.$.

Lemma 2.1. Let $q(\cdot), q_{1}(\cdot) \ldots, q_{m}(\cdot) \in \mathcal{P}_{0}\left(\mathbb{R}^{n}\right)$ so that $1 / q(\cdot)=1 / q_{1}(\cdot)+\cdots+$ $1 / q_{m}(\cdot)$. Then, the inequality

$$
\left\|f_{1} \cdots f_{m}\right\|_{L^{q(\cdot)}\left(\mathbb{R}^{n}\right)} \lesssim\left\|f_{1}\right\|_{L^{q_{1}(\cdot)\left(\mathbb{R}^{n}\right)}} \cdots\left\|f_{m}\right\|_{L^{q_{m}(\cdot)\left(\mathbb{R}^{n}\right)}}
$$

holds for any $f_{j} \in L^{q_{j}(\cdot)}\left(\mathbb{R}^{n}\right), j=1, \ldots, m$.

We define the generalized variable exponent Morrey spaces as follows.

Definition 2.1. Let $p \in \mathcal{P}_{1}\left(\mathbb{R}^{n}\right), \varphi$ be a positive measurable function on $\mathbb{R}^{n} \times$ $(0, \infty)$. We denote by $M^{p(\cdot), \varphi} \equiv M^{p(\cdot), \varphi}\left(\mathbb{R}^{n}\right)$ the generalized variable exponent Morrey space, the space of all functions $f \in L_{\mathrm{loc}}^{p(\cdot)}\left(\mathbb{R}^{n}\right)$ with finite norm

$$
\|f\|_{M^{p(\cdot), \varphi}}=\sup _{x \in \mathbb{R}^{n}, r>0} \varphi(x, r)^{-1} r^{-\theta_{p}(x, r)}\|f\|_{L^{p(\cdot)}(B(x, r))},
$$

where $\|f\|_{L^{p(\cdot)}(B(x, r))} \equiv\left\|f \chi_{B(x, r)}\right\|_{L^{p(\cdot)\left(\mathbb{R}^{n}\right)}}$.

Remark 2.1. Generalized variable exponent Morrey space $M^{p(\cdot), \varphi}$ was introduced and studied by Guliyev, Hasanov and Samko in [19], see also [20].

(1) If $\varphi(x, r)=r^{\frac{\lambda-n}{p(x)}}$ with $0<\lambda<n$, then $M^{p(\cdot), \varphi}=L^{p(\cdot), \lambda}\left(\mathbb{R}^{n}\right)$ is the variable exponent Morrey space introduced by Almeida, Hasanov and Samko in [2].

(2) If $\varphi(x, r) \equiv r^{-\theta_{p}(x, r)}$, then $M^{p(\cdot), \varphi}=L^{p(\cdot)}\left(\mathbb{R}^{n}\right)$ is the variable exponent Lebesgue space.

We will use the following statement on the boundedness of the weighted Hardy operator

$H_{w} g(t):=\int_{t}^{\infty} g(s) w(s) d s, H_{w}^{*} g(t):=\int_{t}^{\infty}\left(1+\ln \frac{s}{t}\right)^{m} g(s) w(s) d s, 0<t<\infty$,

where $w$ is a weight. The following theorem was proved in [18].

Theorem 2.1. [18] Let $v_{1}, v_{2}$ and $w$ be weights on $(0, \infty)$ and $v_{1}(t)$ be bounded outside a neighborhood of the origin. The inequality

$$
\sup _{t>0} v_{2}(t) H_{w} g(t) \leq C \sup _{t>0} v_{1}(t) g(t)
$$

holds for some $C>0$ for all non-negative and non-decreasing $g$ on $(0, \infty)$ if and only if

$$
B:=\sup _{t>0} v_{2}(t) \int_{t}^{\infty} \frac{w(s) d s}{\sup _{s<\tau<\infty} v_{1}(\tau)}<\infty .
$$

Theorem 2.2. [17] Let $v_{1}, v_{2}$ and $w$ be weights on $(0, \infty)$ and $v_{1}(t)$ be bounded outside a neighborhood of the origin. The inequality

$$
\sup _{t>0} v_{2}(t) H_{w}^{*} g(t) \leq C \sup _{t>0} v_{1}(t) g(t)
$$


holds for some $C>0$ for all non-negative and non-decreasing $g$ on $(0, \infty)$ if and only if

$$
B:=\sup _{t>0} v_{2}(t) \int_{t}^{\infty}\left(1+\ln \frac{s}{t}\right)^{m} \frac{w(s) d s}{\sup _{s<\tau<\infty} v_{1}(\tau)}<\infty
$$

\section{3. $\omega$-type Calderón-Zygmund operators in the spaces $M^{p(\cdot), \varphi}$}

The following theorem was proved in [32].

Theorem 3.1. [32] Let $p \in L H\left(\mathbb{R}^{n}\right) \cap \mathcal{P}\left(\mathbb{R}^{n}\right)$ and $T$ be an w-type CalderónZygmund operator defined by (1.2) with $\omega$ satisfying (1.1). Then, the operator $T$ is bounded on $L^{p(\cdot)}\left(\mathbb{R}^{n}\right)$.

The following local estimates are valid (see $[16,20])$.

Theorem 3.2. Let $p \in L H\left(\mathbb{R}^{n}\right) \cap \mathcal{P}\left(\mathbb{R}^{n}\right)$ and $T$ be an $\omega$-type Calderón-Zygmund operator defined by (1.2) with $\omega$ satisfying (1.1). Then the inequality

$$
\|T f\|_{L^{p(\cdot)}(B)} \lesssim r^{\theta_{p}\left(x_{0}, r\right)} \int_{2 r}^{\infty}\|f\|_{L^{p(\cdot)}\left(B\left(x_{0}, t\right)\right)} t^{-\theta_{p}\left(x_{0}, t\right)} \frac{d t}{t}
$$

holds for any ball $B=B\left(x_{0}, r\right)$ and for all $f \in L_{\mathrm{loc}}^{p(\cdot)}\left(\mathbb{R}^{n}\right)$.

Proof. Let $p \in L H\left(\mathbb{R}^{n}\right) \cap \mathcal{P}\left(\mathbb{R}^{n}\right)$. For arbitrary $x_{0} \in \mathbb{R}^{n}$, set $B=B\left(x_{0}, r\right)$ for the ball centered at $x_{0}$ and of radius $r, 2 B=B\left(x_{0}, 2 r\right)$. We represent $f$ as

$$
f=f_{1}+f_{2}, \quad f_{1}(y)=f(y) \chi_{2 B}(y), \quad f_{2}(y)=f(y) \chi_{\mathrm{c}_{(2 B)}}(y), \quad r>0 .
$$

For all $f \in L_{\text {loc }}^{p(\cdot)}\left(\mathbb{R}^{n}\right)$ we define

$$
T f(x):=T_{0} f_{1}(x)+\int_{\mathbb{R}^{n}} K(x, y) f_{2}(y) d y,
$$

here $T_{0}$ denotes a bounded linear operator on $L^{p(\cdot)}$ with $p \in L H\left(\mathbb{R}^{n}\right) \cap \mathcal{P}\left(\mathbb{R}^{n}\right)$ (see $[32,44])$. It is easy to check that the definition of $T f(x)$ does not depend on the choice of the ball $B$. First we show that $\operatorname{Tf}(x)$ is well-defined a.e. $x$ and independent of the choice $B$ containing $x$. As $T_{0}$ is bounded on $L^{p(\cdot)}\left(\mathbb{R}^{n}\right)$ provided by Theorem 3.1 and $f_{1} \in L^{p(\cdot)}\left(\mathbb{R}^{n}\right), T_{0} f_{1}$ is well-defined. Next, we show that the second-term of the right-hand side defining $\operatorname{Tf}(x)$ converges absolutely for any $f \in L_{\text {loc }}^{p(\cdot)}\left(\mathbb{R}^{n}\right)$ and almost every $x \in \mathbb{R}^{n}$.

We have

$$
\|T f\|_{L^{p(\cdot)}(B)} \leq\left\|T f_{1}\right\|_{L^{p(\cdot)}(B)}+\left\|T f_{2}\right\|_{L^{p(\cdot)}(B)} .
$$

Since $f_{1} \in L^{p(\cdot)}\left(\mathbb{R}^{n}\right), T f_{1} \in L^{p(\cdot)}\left(\mathbb{R}^{n}\right)$ and from the boundedness of $T$ in $L^{p(\cdot)}\left(\mathbb{R}^{n}\right)$ (see Theorem 3.1) it follows that

$$
\left\|T f_{1}\right\|_{L^{p(\cdot)}(B)} \leq\left\|T f_{1}\right\|_{L^{p(\cdot)}} \leq C\left\|f_{1}\right\|_{L^{p(\cdot)}}=C\|f\|_{L^{p(\cdot)}(2 B)},
$$

where constant $C>0$ is independent of $f$. 
On the other hand,

$$
\begin{aligned}
\|f\|_{L^{p(\cdot)}(2 B)} & \lesssim|B| \int_{2 r}^{\infty}\|f\|_{L^{p(\cdot)}\left(B\left(x_{0}, t\right)\right)} \frac{d t}{t^{n+1}} \\
& \leq r^{\theta_{p}\left(x_{0}, r\right)}\|1\|_{L^{p^{\prime}}(B)} \int_{2 r}^{\infty}\|f\|_{L^{p(\cdot)}\left(B\left(x_{0}, t\right)\right)} \frac{d t}{t^{n+1}} \\
& \leq r^{\theta_{p}\left(x_{0}, r\right)} \int_{2 r}^{\infty}\|f\|_{L^{p(\cdot)}\left(B\left(x_{0}, t\right)\right)}\|1\|_{L^{p^{\prime}}\left(B\left(x_{0}, t\right)\right)} \frac{d t}{t^{n+1}} \\
& \leq r^{\theta_{p}\left(x_{0}, r\right)} \int_{2 r}^{\infty}\|f\|_{L^{p(\cdot)}\left(B\left(x_{0}, t\right)\right)} t^{-\theta_{p}\left(x_{0}, t\right)} \frac{d t}{t} .
\end{aligned}
$$
have

It is clear that $x \in B, y \in{ }^{\complement}(2 B)$ implies $\frac{1}{2}\left|x_{0}-y\right| \leq|x-y| \leq \frac{3}{2}\left|x_{0}-y\right|$. We

$$
\left|T f_{2}(x)\right| \leq 2^{n} c_{0} \int_{\mathrm{c}_{(2 B)}} \frac{|f(y)|}{\left|x_{0}-y\right|^{n}} d y \lesssim \int_{2 r}^{\infty} \int_{B\left(x_{0}, t\right)}|f(y)| d y \frac{d t}{t^{n+1}} .
$$

Applying Hölder's inequality and from (2.1), we get

$$
\int_{\mathrm{c}_{(2 B)}} \frac{|f(y)|}{\left|x_{0}-y\right|^{n}} d y \lesssim \int_{2 r}^{\infty}\|f\|_{L^{p(\cdot)}\left(B\left(x_{0}, t\right)\right)} t^{-\theta_{p}\left(x_{0}, t\right)} \frac{d t}{t} .
$$

Moreover, for all $p \in L H\left(\mathbb{R}^{n}\right) \cap \mathcal{P}\left(\mathbb{R}^{n}\right)$ the inequality

$$
\left\|T f_{2}\right\|_{L^{p(\cdot)}(B)} \lesssim r^{\theta_{p}\left(x_{0}, r\right)} \int_{2 r}^{\infty}\|f\|_{L^{p(\cdot)}\left(B\left(x_{0}, t\right)\right)} t^{-\theta_{p}\left(x_{0}, t\right)} \frac{d t}{t}
$$

is valid. Thus from (3.3) we have

$$
\begin{aligned}
\|T f\|_{L^{p(\cdot)}(B)} & \lesssim\|f\|_{L^{p(\cdot)}(2 B)}+r^{\theta_{p}\left(x_{0}, r\right)} \int_{2 r}^{\infty}\|f\|_{L^{p(\cdot)}\left(B\left(x_{0}, t\right)\right)} t^{-\theta_{p}\left(x_{0}, t\right)} \frac{d t}{t} \\
& \lesssim r^{\theta_{p}\left(x_{0}, r\right)} \int_{2 r}^{\infty}\|f\|_{L^{p(\cdot)}\left(B\left(x_{0}, t\right)\right)} t^{-\theta_{p}\left(x_{0}, t\right)} \frac{d t}{t} .
\end{aligned}
$$

Then we get the inequality (3.1).

Theorem 3.3. Let $p \in L H\left(\mathbb{R}^{n}\right) \cap \mathcal{P}\left(\mathbb{R}^{n}\right)$, $T$ be an $\omega$-type Calderón-Zygmund operator defined by (1.2) with $\omega$ satisfying $(1.1)$, and $\left(\varphi_{1}, \varphi_{2}\right)$ satisfy the condition

$$
\int_{r}^{\infty} \frac{\underset{t<s<\infty}{\operatorname{essinf}} \varphi_{1}(x, s) s^{\theta_{p}(x, s)}}{t^{\theta_{p}(x, t)}} \frac{d t}{t} \leq C \varphi_{2}(x, r)
$$

where $C$ does not depend on $x$ and $r$. Then the operator $T$ is bounded from $M^{p(\cdot), \varphi_{1}}$ to $M^{p(\cdot), \varphi_{2}}$.

Proof. For $p \in L H\left(\mathbb{R}^{n}\right) \cap \mathcal{P}\left(\mathbb{R}^{n}\right)$ from Theorem 2.1 and Theorem 3.2 we get

$$
\begin{aligned}
\|T f\|_{M^{p(\cdot), \varphi_{2}}} & \lesssim \sup _{x \in \mathbb{R}^{n}, r>0} \varphi_{2}(x, r)^{-1} \int_{r}^{\infty}\|f\|_{L^{p(\cdot)}(B(x, t))} t^{-\theta_{p}(x, t)} \frac{d t}{t} \\
& \lesssim \sup _{x \in \mathbb{R}^{n}, r>0} \varphi_{1}(x, r)^{-1} r^{-\theta_{p}(x, r)}\|f\|_{L^{p(\cdot)(B)}}=\|f\|_{M^{p(\cdot), \varphi_{1}}} .
\end{aligned}
$$

In the case $p(x) \equiv$ const from Theorem 3.3 we get the following corollary, which was proved in [25]. 
Corollary 3.1. [25] Let $T$ be an $\omega$-type Calderón-Zygmund operator defined by (1.2) with $\omega$ satisfying (1.1). Let also $1<p<\infty$ and $\left(\varphi_{1}, \varphi_{2}\right)$ satisfy the condition

$$
\int_{r}^{\infty} \frac{\underset{t<s<\infty}{\operatorname{essinf}} \varphi_{1}(x, s) s^{\frac{n}{p}}}{t^{\frac{n}{p}}} \frac{d t}{t} \leq C \varphi_{2}(x, r)
$$

where $C$ does not depend on $x$ and $r$. Then the operator $T$ is bounded from $M^{p, \varphi_{1}}$ to $M^{p, \varphi_{2}}$.

Remark 3.1. Let $0 \leq \kappa<1$. Assume that $\psi$ is a positive increasing function defined in $(0, \infty)$ and satisfies the following $\mathcal{D}_{\kappa}$ condition :

$$
\frac{\psi\left(t_{2}\right)}{t_{2}^{\kappa}} \leq C \frac{\psi\left(t_{1}\right)}{t_{1}^{\kappa}}, \text { for any } 0<t_{1}<t_{2}<\infty
$$

where $C>0$ is a constant independent of $t_{1}$ and $t_{2}$. If $p(x) \equiv$ const, $\varphi_{1}(x, r)=$ $\varphi_{2}(x, r)=\psi(w(x, r))$ and $\psi$ satisfy the $\mathcal{D}_{\kappa}$ condition, Theorems 3.2 and 3.3 were proved in [41]. Also, in the case $\omega(t)=t^{\varepsilon}$ with $0<\varepsilon \leq 1$, Theorems 3.2 and 3.3 were proved in [19].

\section{Commutators of $\omega$-type Calderón-Zygmund operators in the spaces $M^{p(\cdot), \varphi}$}

We recall the definition of the space of $B M O\left(\mathbb{R}^{n}\right)$.

Definition 4.1. Suppose that $b \in L_{1}^{\text {loc }}\left(\mathbb{R}^{n}\right)$, and let

$$
\|b\|_{*}=\sup _{x \in \mathbb{R}^{n}, r>0} \frac{1}{|B(x, r)|} \int_{B(x, r)}\left|b(y)-b_{B(x, r)}\right| d y<\infty,
$$

where $b_{B(x, r)}=\frac{1}{|B(x, r)|} \int_{B(x, r)} b(y) d y$. Define

$$
B M O\left(\mathbb{R}^{n}\right)=\left\{b \in L_{1}^{\text {loc }}\left(\mathbb{R}^{n}\right):\|b\|_{*}<\infty\right\} .
$$

Modulo constants, the space $B M O\left(\mathbb{R}^{n}\right)$ is a Banach space with respect to the norm $\|\cdot\|_{*}$.

By the generalized Hölder's inequality in Orlicz spaces (see [37, page 58]) and John-Nirenberg's inequality, we get (see also $[28,(2.14)]$ )

$$
\frac{1}{|B|} \int_{B}\left|b_{1}(x)-\left(b_{1}\right)_{B}\right| \ldots\left|b_{m}(x)-\left(b_{m}\right)_{B}\right||g(x)| d x \lesssim \prod_{j=1}^{m}\left\|b_{j}\right\|_{B M O}\|g\|_{L(\log L)^{m}, B}
$$

Definition 4.2. The $B M O_{p(\cdot)}\left(\mathbb{R}^{n}\right)$ space is the set of all locally integrable functions $f$ with finite norm

$$
\|b\|_{B M O_{p(\cdot)}}=\sup _{B} \frac{\left\|\left(b(\cdot)-b_{B}\right) \chi_{B}\right\|_{L^{p(\cdot)}}}{\left\|\chi_{B}\right\|_{L^{p(\cdot)}}} .
$$

Theorem 4.1. [23] Let $p \in L H\left(\mathbb{R}^{n}\right) \cap \mathcal{P}\left(\mathbb{R}^{n}\right)$. Then, the norms $\|\cdot\|_{B M O_{p(\cdot)}}$ and $\|\cdot\|_{B M O}$ are mutually equivalent. 
Since linear commutator has a greater degree of singularity than the corresponding $\omega$-type Calderón-Zygmund operator, we need a slightly stronger version of condition

$$
\int_{0}^{1} \frac{\omega(t)}{t}\left(1+\log \frac{1}{t}\right)^{m} d t<\infty .
$$

The following weighted endpoint estimate for commutator $T_{\vec{b}}$ of the $\omega$-type Calderón-Zygmund operator was established in [44] under a stronger version of condition (4.2) imposed on $\omega$, if $\vec{b} \in B M O^{m}\left(\mathbb{R}^{n}\right)$ (for the unweighted case, see [31]).

The following theorem was proved in $[40,44]$.

Theorem 4.2. [40,44] Let $T$ be a linear $\omega-C Z O$ and $\vec{b} \in B M O^{m}\left(\mathbb{R}^{n}\right)$. If $\omega$ satisfies condition (4.2) and $p \in L H\left(\mathbb{R}^{n}\right) \cap \mathcal{P}\left(\mathbb{R}^{n}\right)$, then there exists a constant $C>0$ such that

$$
\left\|T_{\vec{b}} f\right\|_{L^{p(\cdot)}} \leq C\|\vec{b}\|_{*}\|f\|_{L^{p(\cdot)}},
$$

where $\|\vec{b}\|_{*}=\prod_{j=1}^{m}\left\|b_{j}\right\|_{*}$.

The following local estimates are valid (see [17]).

Theorem 4.3. Let $T$ be a linear $\omega$-CZO and $\vec{b} \in B M O^{m}\left(\mathbb{R}^{n}\right)$. Let also $\omega$ satisfying condition (4.2) and $p \in L H\left(\mathbb{R}^{n}\right) \cap \mathcal{P}\left(\mathbb{R}^{n}\right)$. Then

$$
\left\|T_{\vec{b}} f\right\|_{L^{p(\cdot)}(B)} \leq C\|\vec{b}\|_{*} r^{\theta_{p}\left(x_{0}, r\right)} \int_{2 r}^{\infty} \ln ^{m}\left(e+\frac{t}{r}\right)\|f\|_{L^{p(\cdot)}\left(B\left(x_{0}, t\right)\right)} t^{-\theta_{p}\left(x_{0}, t\right)} \frac{d t}{t}
$$

holds for any ball $B=B\left(x_{0}, r\right)$ and for all $f \in L_{\mathrm{loc}}^{p(\cdot)}\left(\mathbb{R}^{n}\right)$, where $C$ does not depend on $f, x_{0} \in \mathbb{R}^{n}$ and $r>0$.

Proof. Let $p \in L H\left(\mathbb{R}^{n}\right) \cap \mathcal{P}\left(\mathbb{R}^{n}\right)$. For arbitrary $x_{0} \in \mathbb{R}^{n}$ and $r>0$, set $B=$ $B\left(x_{0}, r\right)$. Write $f=f_{1}+f_{2}$ with $f_{1}=f \chi_{2 B}$ and $f_{2}=f \chi_{\mathrm{c}_{(2 B)}}$. For all $f \in$ $L_{\text {loc }}^{p(\cdot)}\left(\mathbb{R}^{n}\right)$ we define

$$
T_{\vec{b}} f(x):=T_{\vec{b}, 0} f_{1}(x)+\int_{\mathbb{R}^{n}} \prod_{j=1}^{m}\left(b_{j}(x)-b_{j}(y)\right) K(x, y) f_{2}(y) d y,
$$

here $T_{\vec{b}, 0}$ denotes the commutator as a bounded linear operator on $L^{p(\cdot)}$ with $p \in L H\left(\mathbb{R}^{n}\right) \cap \mathcal{P}\left(\mathbb{R}^{n}\right)$ (see [44]). It is easy to check that the definition of $T_{\vec{b}} f(x)$ does not depend on the choice of the ball $B$. First we show that $T_{\vec{b}} f(x)$ is well-defined a.e. $x$ and independent of the choice $B$ containing $x$. As $T_{\vec{b}, 0}$ is bounded on $L^{p(\cdot)}\left(\mathbb{R}^{n}\right)$ provided by Theorem 4.2 and $f_{1} \in L^{p(\cdot)}\left(\mathbb{R}^{n}\right), T_{\vec{b}, 0} f_{1}$ is well-defined. Next, we show that the second-term of the right-hand side defining $T f(x)$ converges absolutely for any $f \in L_{\text {loc }}^{p(\cdot)}\left(\mathbb{R}^{n}\right)$ and almost every $x \in \mathbb{R}^{n}$.

Hence

$$
\left\|T_{\vec{b}} f\right\|_{L^{p(\cdot)}(B)} \leq\left\|T_{\vec{b}} f_{1}\right\|_{L^{p(\cdot)}(B)}+\left\|T_{\vec{b}} f_{2}\right\|_{L^{p(\cdot)}(B)} .
$$

From the boundedness of $T_{\vec{b}}$ in $L^{p(\cdot)}\left(\mathbb{R}^{n}\right)$ ( see Theorem 4.2) it follows that:

$$
\left\|T_{\vec{b}} f_{1}\right\|_{L^{p(\cdot)}(B)} \leq\left\|T_{\vec{b}} f_{1}\right\|_{L^{p(\cdot)}} \lesssim\|\vec{b}\|_{*}\left\|f_{1}\right\|_{L^{p(\cdot)}}=\|\vec{b}\|_{*}\|f\|_{L^{p(\cdot)}(2 B)} .
$$


For the term $\left\|T_{\vec{b}} f_{2}\right\|_{L^{p(\cdot)(B)}}$, without loss of generality, we can assume $m=2$. Thus, the operator $T_{\vec{b}} f_{2}$ can be divided into four parts

$$
\begin{aligned}
T_{\vec{b}} f_{2}(x) & =\left(b_{1}(x)-\left(b_{1}\right)_{B}\right)\left(b_{2}(x)-\left(b_{2}\right)_{B}\right) \int_{\mathbb{R}^{n}} K(x, y) f_{2}(y) d y \\
& +\int_{\mathbb{R}^{n}} K(x, y)\left(b_{1}(y)-\left(b_{1}\right)_{B}\right)\left(b_{2}(y)-\left(b_{2}\right)_{B}\right) f_{2}(y) d y \\
& -\left(b_{1}(x)-\left(b_{1}\right)_{B}\right) \int_{\mathbb{R}^{n}} K(x, y)\left(b_{2}(y)-\left(b_{2}\right)_{B}\right) f_{2}(y) d y \\
& -\left(b_{2}(x)-\left(b_{2}\right)_{B}\right) \int_{\mathbb{R}^{n}} K(x, y)\left(b_{1}(y)-\left(b_{1}\right)_{B}\right) f_{2}(y) d y \\
& =I_{1}(x)+I_{2}(x)+I_{3}(x)+I_{4}(x) .
\end{aligned}
$$

For $x \in B$ we have

$$
\begin{aligned}
\left|T_{\vec{b}} f_{2}(x)\right| & \leq\left|I_{1}(x)+\right| I_{2}(x)|+| I_{3}(x)|+| I_{4}(x) \mid \\
& \lesssim\left|b_{1}(x)-\left(b_{1}\right)_{B}\right|\left|b_{2}(x)-\left(b_{2}\right)_{B}\right| \int_{\mathrm{c}_{(2 B)}} \frac{|f(y)|}{\left|x_{0}-y\right|^{n}} d y \\
& +\int_{\mathrm{c}_{(2 B)}}\left|b_{1}(y)-\left(b_{1}\right)_{B}\right|\left|b_{2}(y)-\left(b_{2}\right)_{B}\right| \frac{|f(y)|}{\left|x_{0}-y\right|^{n}} d y \\
& +\left|b_{1}(x)-\left(b_{1}\right)_{B}\right| \int_{\mathrm{c}_{(2 B)}}\left|b_{2}(y)-\left(b_{2}\right)_{B}\right| \frac{|f(y)|}{\left|x_{0}-y\right|^{n}} d y \\
& +\left|b_{2}(x)-\left(b_{2}\right)_{B}\right| \int_{\mathrm{C}_{(2 B)}}\left|b_{1}(y)-\left(b_{1}\right)_{B}\right| \frac{|f(y)|}{\left|x_{0}-y\right|^{n}} d y .
\end{aligned}
$$

Then

$$
\begin{aligned}
&\left\|T_{\vec{b}} f_{2}\right\|_{L^{p(\cdot)}(B)} \lesssim\left\|\int_{\mathrm{c}_{(2 B)}} \frac{\prod_{j=1}^{2}\left|b_{i}(y)-\left(b_{i}\right)_{B}\right|}{\left|x_{0}-y\right|^{n}}|f(y)| d y\right\|_{L^{p(\cdot)}(B)} \\
&+\left\|\left|b_{1}(x)-\left(b_{1}\right)_{B}\right| \int_{\mathrm{c}_{(2 B)}} \frac{\left|b_{2}(y)-\left(b_{2}\right)_{B}\right|}{\left|x_{0}-y\right|^{n}}|f(y)| d y\right\|_{L^{p(\cdot)}(B)} \\
&+\left\|\left|b_{2}(x)-\left(b_{2}\right)_{B}\right| \int_{\mathrm{c}_{(2 B)}} \frac{\left|b_{1}(y)-\left(b_{1}\right)_{B}\right|}{\left|x_{0}-y\right|^{n}}|f(y)| d y\right\|_{L^{p(\cdot)}(B)} \\
& \\
&+\left\|\int_{\mathrm{c}_{(2 B)}} \frac{\prod_{j=1}^{2}\left|b_{i}(x)-\left(b_{i}\right)_{B}\right|}{\left|x_{0}-y\right|^{n}}|f(y)| d y\right\|_{L^{p(\cdot)}(B)} \\
&=I_{1}+I_{2}+I_{3}+I_{4} .
\end{aligned}
$$

Let us estimate $I_{1}$. 


$$
\begin{aligned}
I_{1} & =r^{\theta_{p}\left(x_{0}, r\right)} \int_{\mathrm{C}_{(2 B)}} \frac{\prod_{j=1}^{2}\left|b_{i}(y)-\left(b_{i}\right)_{B}\right|}{\left|x_{0}-y\right|^{n}}|f(y)| d y \\
& \approx r^{\theta_{p}\left(x_{0}, r\right)} \int_{\mathrm{C}_{(2 B)}} \prod_{j=1}^{2}\left|b_{i}(y)-\left(b_{i}\right)_{B}\right||f(y)| \int_{\left|x_{0}-y\right|}^{\infty} \frac{d t}{t^{n+1}} d y \\
& \approx r^{\theta_{p}\left(x_{0}, r\right)} \int_{2 r}^{\infty} \int_{2 r \leq\left|x_{0}-y\right| \leq t} \prod_{j=1}^{2}\left|b_{i}(y)-\left(b_{i}\right)_{B}\right||f(y)| d y \frac{d t}{t^{n+1}} \\
& \lesssim r^{\theta_{p}\left(x_{0}, r\right)} \int_{2 r}^{\infty} \int_{B\left(x_{0}, t\right)} \prod_{j=1}^{2}\left|b_{i}(y)-\left(b_{i}\right)_{B}\right||f(y)| d y \frac{d t}{t^{n+1}} .
\end{aligned}
$$

Applying Hölder's inequality (4.1), by Lemma 2.1 and Theorem 4.1, we get

$$
\begin{aligned}
& I_{1} \lesssim r^{\theta_{p}\left(x_{0}, r\right)} \int_{2 r}^{\infty}\left\|\prod_{j=1}^{2}\left|b_{i}(\cdot)-\left(b_{i}\right)_{B}\right|\right\|_{L^{p^{\prime}(\cdot)\left(B\left(x_{0}, t\right)\right)}}\|f\|_{L^{p(\cdot)\left(B\left(x_{0}, t\right)\right)}} \frac{d t}{t^{n+1}} \\
& \lesssim r^{\theta_{p}\left(x_{0}, r\right)}\left(\int_{2 r}^{\infty}\left\|\prod_{j=1}^{2}\left|b_{i}(\cdot)-\left(b_{i}\right)_{B\left(x_{0}, t\right)}\right|\right\|_{L^{p^{(\cdot)}\left(B\left(x_{0}, t\right)\right)}}\|f\|_{L^{p(\cdot)}\left(B\left(x_{0}, t\right)\right)} \frac{d t}{t^{n+1}}\right. \\
& +\int_{2 r}^{\infty}\left|\left(b_{1}\right)_{B\left(x_{0}, t\right)}-\left(b_{1}\right)_{B}\right|\left\|b_{2}(\cdot)-\left(b_{2}\right)_{B\left(x_{0}, t\right)}\right\|_{L^{p^{\prime}(\cdot)\left(B\left(x_{0}, t\right)\right)}}\|f\|_{L^{p(\cdot)}\left(B\left(x_{0}, t\right)\right)} \frac{d t}{t^{n+1}} \\
& +\int_{2 r}^{\infty}\left|\left(b_{2}\right)_{B\left(x_{0}, t\right)}-\left(b_{2}\right)_{B}\right|\left\|b_{1}(\cdot)-\left(b_{1}\right)_{B\left(x_{0}, t\right)}\right\|_{L^{p^{\prime}(\cdot)\left(B\left(x_{0}, t\right)\right)}}\|f\|_{L^{p(\cdot)}\left(B\left(x_{0}, t\right)\right)} \frac{d t}{t^{n+1}} \\
& \left.+\int_{2 r}^{\infty} \prod_{j=1}^{2}\left|\left(b_{i}\right)_{B\left(x_{0}, t\right)}-\left(b_{i}\right)_{B}\right|\|1\|_{L^{p^{\prime}(\cdot)}\left(B\left(x_{0}, t\right)\right)}\|f\|_{L^{p(\cdot)}\left(B\left(x_{0}, t\right)\right)} \frac{d t}{t^{n+1}}\right) \\
& \lesssim r^{\theta_{p}\left(x_{0}, r\right)} \int_{2 r}^{\infty} \prod_{j=1}^{2}\left\|b_{i}(\cdot)-\left(b_{i}\right)_{B\left(x_{0}, t\right)}\right\|_{L^{2 p^{\prime}(\cdot)\left(B\left(x_{0}, t\right)\right)}}\|f\|_{L^{p(\cdot)}\left(B\left(x_{0}, t\right)\right)} \frac{d t}{t^{n+1}} \\
& +\|\vec{b}\|_{*} r^{\theta_{p}\left(x_{0}, r\right)} \int_{2 r}^{\infty} \ln \frac{t}{r}\|1\|_{L^{2 p^{\prime}(\cdot)\left(B\left(x_{0}, t\right)\right)}}\|f\|_{L^{p(\cdot)}\left(B\left(x_{0}, t\right)\right)} \frac{d t}{t^{n+1}} \\
& +r^{\theta_{p}\left(x_{0}, r\right)} \int_{2 r}^{\infty} \prod_{j=1}^{2}\left|\left(b_{i}\right)_{B\left(x_{0}, t\right)}-\left(b_{i}\right)_{B}\right|\|1\|_{L^{p^{\prime}(\cdot)\left(B\left(x_{0}, t\right)\right)}}\|f\|_{L^{p(\cdot)}\left(B\left(x_{0}, t\right)\right)} \frac{d t}{t^{n+1}} \\
& \lesssim\|\vec{b}\|_{*} r^{\theta_{p}\left(x_{0}, r\right)} \int_{2 r}^{\infty} \ln ^{2}\left(e+\frac{t}{r}\right)\|1\|_{L^{p^{\prime}}\left(B\left(x_{0}, t\right)\right)}\|f\|_{L^{p(\cdot)}\left(B\left(x_{0}, t\right)\right)} \frac{d t}{t^{n+1}} \\
& \lesssim\|\vec{b}\|_{*} r^{\theta_{p}\left(x_{0}, r\right)} \int_{2 r}^{\infty} \ln ^{2}\left(e+\frac{t}{r}\right)\|f\|_{L^{p(\cdot)}\left(B\left(x_{0}, t\right)\right)} t^{-\theta_{p}\left(x_{0}, t\right)} \frac{d t}{t} .
\end{aligned}
$$

Let us estimate $I_{2}$. 


$$
\begin{aligned}
I_{2} & =\left\|b_{1}(\cdot)-\left(b_{1}\right)_{B}\right\|_{L^{p(\cdot)}\left(B\left(x_{0}, t\right)\right)} \int_{\mathrm{c}_{(2 B)}} \frac{\left|b_{2}(y)-\left(b_{2}\right)_{B}\right|}{\left|x_{0}-y\right|^{n}}|f(y)| d y \\
& \lesssim\left\|b_{1}\right\|_{*} r^{\theta_{p}\left(x_{0}, r\right)} \int_{\mathrm{c}_{(2 B)}}\left|b_{2}(y)-\left(b_{2}\right)_{B}\right||f(y)| \int_{\left|x_{0}-y\right|}^{\infty} \frac{d t}{t^{n+1}} d y \\
& \approx\left\|b_{1}\right\|_{*} r^{\theta_{p}\left(x_{0}, r\right)} \int_{2 r}^{\infty} \int_{2 r \leq\left|x_{0}-y\right| \leq t}\left|b_{2}(y)-\left(b_{2}\right)_{B}\right||f(y)| d y \frac{d t}{t^{n+1}} \\
& \lesssim\left\|b_{1}\right\|_{*} r^{\theta_{p}\left(x_{0}, r\right)} \int_{2 r}^{\infty} \int_{B\left(x_{0}, t\right)}\left|b_{2}(y)-\left(b_{2}\right)_{B}\right||f(y)| d y \frac{d t}{t^{n+1}} .
\end{aligned}
$$

Applying Hölder's inequality (4.1) and by Theorem 4.1, we get

$$
\begin{aligned}
I_{2} & \lesssim\left\|b_{1}\right\|_{*} r^{\theta_{p}\left(x_{0}, r\right)} \int_{2 r}^{\infty}\left\|b_{2}(\cdot)-\left(b_{2}\right)_{B}\right\|_{L^{p^{\prime}(\cdot)\left(B\left(x_{0}, t\right)\right)}}\|f\|_{L^{p(\cdot)}\left(B\left(x_{0}, t\right)\right)} \frac{d t}{t^{n+1}} \\
& \lesssim \prod_{j=1}^{2}\left\|b_{j}\right\|_{*} r^{\theta_{p}\left(x_{0}, r\right)} \int_{2 r}^{\infty}\left(1+\ln \frac{t}{r}\right)\|1\|_{L^{p^{\prime}\left(B\left(x_{0}, t\right)\right)}}\|f\|_{L^{p(\cdot)}\left(B\left(x_{0}, t\right)\right)} \frac{d t}{t^{n+1}} \\
& \lesssim\|\vec{b}\|_{*} r^{\theta_{p}\left(x_{0}, r\right)} \int_{2 r}^{\infty} \ln ^{2}\left(e+\frac{t}{r}\right)\|f\|_{L^{p(\cdot)}\left(B\left(x_{0}, t\right)\right)} t^{-\theta_{p}\left(x_{0}, t\right)} \frac{d t}{t} .
\end{aligned}
$$

In the same way, we shall get the result of $I_{3}$

$$
I_{3} \lesssim\|\vec{b}\|_{*} r^{\theta_{p}\left(x_{0}, r\right)} \int_{2 r}^{\infty} \ln ^{2}\left(e+\frac{t}{r}\right)\|f\|_{L^{p(\cdot)}\left(B\left(x_{0}, t\right)\right)} t^{-\theta_{p}\left(x_{0}, t\right)} \frac{d t}{t} .
$$

In order to estimate $I_{4}$ we note that

$$
\begin{aligned}
I_{4} & =\left\|\prod_{j=1}^{2} \mid b_{i}(x)-\left(b_{i}\right)_{B}\right\|_{L^{p(\cdot)}\left(B\left(x_{0}, t\right)\right)} \int_{\mathrm{c}_{(2 B)}} \frac{|f(y)|}{\left|x_{0}-y\right|^{n}} d y \\
& \leq \prod_{j=1}^{2}\left\|b_{i}(x)-\left(b_{i}\right)_{B}\right\|_{L^{2 p(\cdot)\left(B\left(x_{0}, t\right)\right)}} \int_{\mathrm{c}_{(2 B)}} \frac{|f(y)|}{\left|x_{0}-y\right|^{n}} d y .
\end{aligned}
$$

By Theorem 4.1, we get

$$
I_{4} \lesssim\|\vec{b}\|_{*} r^{\theta_{p}\left(x_{0}, r\right)} \int_{\mathrm{C}_{(2 B)}} \frac{|f(y)|}{\left|x_{0}-y\right|^{n}} d y
$$

Thus, by (3.4)

$$
I_{4} \lesssim\|\vec{b}\|_{*} r^{\theta_{p}\left(x_{0}, r\right)} \int_{2 r}^{\infty}\|f\|_{L^{p(\cdot)}\left(B\left(x_{0}, t\right)\right)} t^{-\theta_{p}\left(x_{0}, t\right)} \frac{d t}{t} .
$$

Summing up $I_{1}$ and $I_{4}$, for all $p \in L H\left(\mathbb{R}^{n}\right) \cap \mathcal{P}\left(\mathbb{R}^{n}\right)$ we get

$$
\left\|T_{\vec{b}} f_{2}\right\|_{L^{p(\cdot)}(B)} \lesssim\|\vec{b}\|_{*} r^{\theta_{p}\left(x_{0}, r\right)} \int_{2 r}^{\infty} \ln ^{2}\left(e+\frac{t}{r}\right)\|f\|_{L^{p(\cdot)}\left(B\left(x_{0}, t\right)\right)} t^{-\theta_{p}\left(x_{0}, t\right)} \frac{d t}{t} .
$$


Finally,

$$
\begin{aligned}
\left\|T_{\vec{b}} f\right\|_{L^{p(\cdot)}(B)} & \lesssim\|\vec{b}\|_{*}\|f\|_{L^{p(\cdot)}(2 B)} \\
& +\|\vec{b}\|_{*} r^{\theta_{p}\left(x_{0}, r\right)} \int_{2 r}^{\infty} \ln ^{m}\left(e+\frac{t}{r}\right)\|f\|_{L^{p(\cdot)}\left(B\left(x_{0}, t\right)\right)} t^{-\theta_{p}\left(x_{0}, t\right)} \frac{d t}{t},
\end{aligned}
$$

and the statement of Theorem 4.3 follows by (3.3).

Theorem 4.4. Let $T$ be a linear $\omega-C Z O$ and $\vec{b} \in B M O^{m}\left(\mathbb{R}^{n}\right)$. Let also $\omega$ satisfying condition (4.2), $p \in L H\left(\mathbb{R}^{n}\right) \cap \mathcal{P}\left(\mathbb{R}^{n}\right)$ and $\left(\varphi_{1}, \varphi_{2}\right)$ satisfy the condition

$$
\int_{r}^{\infty} \ln ^{m}\left(e+\frac{t}{r}\right) \frac{\underset{t<s<\infty}{\operatorname{essinf}} \varphi_{1}(x, s) s^{\theta_{p}(x, s)}}{t^{\theta_{p}(x, t)}} \frac{d t}{t} \leq C \varphi_{2}(x, r),
$$

where $C$ does not depend on $x$ and $r$. Then the operator $T_{\vec{b}}$ is bounded from $M^{p(\cdot), \varphi_{1}}$ to $M^{p(\cdot), \varphi_{2}}$. Moreover,

$$
\left\|T_{\vec{b}} f\right\|_{M^{p(\cdot), \varphi_{2}}} \lesssim\|\vec{b}\|_{*}\|f\|_{M^{p(\cdot), \varphi_{1}}} .
$$

Proof. Using Theorem 2.2 and Theorem 4.3 we have

$$
\begin{aligned}
& \left\|T_{\vec{b}} f\right\|_{M^{p(\cdot), \varphi_{2}}}=\sup _{x \in \mathbb{R}^{n}, r>0} \varphi_{2}(x, r)^{-1} r^{-\theta_{p}(x, r)}\left\|T_{\vec{b}} f\right\|_{L^{p(\cdot)} B(x, r)} \\
& \lesssim\|\vec{b}\|_{*} \sup _{x \in \mathbb{R}^{n}, r>0} \varphi_{2}(x, r)^{-1} \int_{r}^{\infty} \ln ^{m}\left(e+\frac{t}{r}\right)\|f\|_{L^{p(\cdot)}(B(x, t))} t^{-\theta_{p}(x, t)} \frac{d t}{t} \\
& \lesssim\|\vec{b}\|_{*} \sup _{x \in \mathbb{R}^{n}, r>0} \varphi_{1}(x, r)^{-1} r^{-\theta_{p}(x, r)}\|f\|_{L^{p(\cdot)}(B(x, r))}=\|\vec{b}\|_{*}\|f\|_{M^{p(\cdot), \varphi_{1}}}
\end{aligned}
$$

In the case $p(x) \equiv$ const from Theorem 4.4 we get the following corollary, which was proved in [25].

Corollary 4.1. [25] Let $T$ be a linear $\omega$-CZO and $\vec{b} \in B M O^{m}\left(\mathbb{R}^{n}\right)$. Let also $\omega$ satisfy condition (4.2), $1<p<\infty$ and $\left(\varphi_{1}, \varphi_{2}\right)$ satisfy the condition

$$
\int_{r}^{\infty} \ln ^{m}\left(e+\frac{t}{r}\right) \frac{\underset{t<s<\infty}{\operatorname{essinf}} \varphi_{1}(x, s) s^{\frac{n}{p}}}{t^{\frac{n}{p}}} \frac{d t}{t} \leq C \varphi_{2}(x, r),
$$

where $C$ does not depend on $x$ and $r$. Then the operator $T_{\vec{b}}$ is bounded from $M^{p, \varphi_{1}}$ to $M^{p, \varphi_{2}}$.

Remark 4.1. Note that, if $p(x) \equiv$ const, $\varphi_{1}(x, r)=\varphi_{2}(x, r)=\psi(w(x, r))$ and $\psi$ satisfy the $\mathcal{D}_{\kappa}$ condition, Corollary 4.1 were proved in [41]. Also, in the case $\omega(t)=t^{\varepsilon}$ with $0<\varepsilon \leq 1$, Theorems 4.3 and 4.4 were proved in [11], and in the case $m=1$ in [22].

Acknowledgments. The research of V. Guliyev was supported by the grant of Cooperation Program 2532 TUBITAK-RFBR (RUSSIAN foundation for basic research) (Agreement number no. 119N455) and by the Grant of 1st AzerbaijanRussia Joint Grant Competition (Agreement Number No. EIF-BGM-4-RFTF1/2017-21/01/1-M-08). 


\section{References}

[1] A. Akbulut, X.A. Badalov, J.J. Hasanov, A. Serbetci, $p(x)$-admissible sublinear singular operators in the generalized variable exponent Morrey spaces, Trans. Natl. Acad. Sci. Azerb. Ser. Phys.-Tech. Math. Sci. 36 (1) (2016), Mathematics, 10-17.

[2] A. Almeida, J. Hasanov, S. Samko, Maximal and potential operators in variable exponent Morrey spaces, Georgian Math. J. 15 (2) (2008), 195-208.

[3] A.P. Calderón, Commutators of singular integral operators, Proc. Natl. Acad. Sci. USA 53 (1965), 1092-1099.

[4] A.P. Calderón, Cauchy integrals on Lipschitz curves and related operators, Proc. Natl. Acad. Sci. USA 74 (1977), no. 4, 1324-1327.

[5] F. Chiarenza, M. Frasca, P. Longo, $W^{2, p}$-solvability of Dirichlet problem for nondivergence ellipic equations with VMO coefficients, Trans. Amer. Math. Soc. 336 (1993), 841-853.

[6] R.R. Coifman, Y. Meyer, Au delà des Opérateurs Pseudo-Différentiels, Astérisque 57. Société Maématique de France, Paris, France, 1978.

[7] D. Cruz-Uribe, A. Fiorenza, J. Martell, C. Perez, The boundedness of classical operators on variable $L^{p}$ spaces, Ann. Acad. Sci. Fenn. Math. 31 (2006), 239-264.

[8] D. Cruz-Uribe, A. Fiorenza, Variable Lebesgue Spaces, Foundations and Harmonic Analysis, 1st edn. Birkhauser, Basel, 2013.

[9] L. Diening and M. Ružicka, Calderón-Zygmund operators on generalized Lebesgue spaces $L^{p(\cdot)}$ and problems related to fluid dynamics, J. Reine Angew Math. 563 (2003), 197-220.

[10] L. Diening, P. Harjulehto, Hästö and M. Ružička, Lebesgue and Sobolev spaces with variable exponents, Springer-Verlag, Lecture Notes in Mathematics, vol. 2017, Berlin, 2011.

[11] I. Ekincioglu, C. Keskin, A. Serbetci, Multilinear commutators of CalderónZygmund operator on generalized variable exponent Morrey spaces. Positivity (2021). https://doi.org/10.1007/s11117-021-00828-3

[12] A. Eroglu, M.N. Omarova, Sh.A. Muradova, Elliptic equations with measurable coefficients in generalized weighted Morrey spaces, Proc. Inst. Math. Mech. Natl. Acad. Sci. Azerb. 43 (2017), no. 2, 197-213.

[13] X. Fan, Variable exponent Morrey and Campanato spaces, Nonlinear Anal. 72 (2010), no. 11, 4148-4161.

[14] G. Di Fazio, D.I. Hakim, Y. Sawano, Elliptic equations with discontinuous coefficients in generalized Morrey spaces, Eur. J. Math. 3 (2017), no. 3, 728-762.

[15] V.S. Guliyev, Integral operators on function spaces on the homogeneous groups and on domains in $\mathbb{R}^{n}$, Doctoral dissertation, Moscow, Mat. Inst. Steklov, 1994, 329 pp. (in Russian)

[16] V.S. Guliyev, Boundedness of the maximal, potential and singular operators in the generalized Morrey spaces, J. Inequal. Appl. (2009), Art. ID 503948, 20 pp.

[17] V.S. Guliyev, Generalized weighted Morrey spaces and higher order commutators of sublinear operators, Eurasian Math. J. 3 (2012), no. 3, 33-61.

[18] V.S. Guliyev, Generalized local Morrey spaces and fractional integral operators with rough kernel, J. Math. Sci. (N. Y.) 193 (2013), no. 2, 211-227.

[19] V.S. Guliyev, J. Hasanov, S. Samko, Boundedness of the maximal, potential and singular operators in the generalized variable exponent Morrey spaces, Math. Scand., 107 (2) (2010), 285-304.

[20] V.S. Guliyev, S.G. Samko, Maximal, potential and singular operators in the generalized variable exponent Morrey spaces on unbounded sets, J. Math. Sci. (N.Y.) 193 (2) (2013), 228-248. 
[21] V.S. Guliev, S.G. Samko, Maximal operator in variable exponent generalized Morrey spaces on quasi-metric measure space, Mediterr. J. Math. 13 (3) (2016), 1151-1165.

[22] J.J. Hasanov, S.S. Aliyev, S. Seymur, Y.Y. Guliyev, Commutators of potential and singular integral operators in generalized variable exponent Morrey spaces, Trans. Natl. Acad. Sci. Azerb. Ser. Phys.-Tech. Math. Sci. 35 (2015), no. 4, Mathematics, 75-83.

[23] Kwok-Pun Ho, Singular integral operators, John-Nirenberg inequalities and TribelLizorkin type spaces on weighted Lebesgue spaces with variable exponents, Rev. Un. Mat. Argentina 57 (2016), no. 1, 85-101.

[24] Aiwu Huang, Jingshi Xu, Multilinear singular integrals and commutators in variable exponent Lebesgue spaces, Appl. Math. J. Chinese Univ. 25 B (1), 2010, 69-77.

[25] A.F. Ismayilova, Calderón-Zygmund operators with kernels of Dini's type and their multilinear commutators on generalized Morrey spaces, Trans. Natl. Acad. Sci. Azerb. Ser. Phys.-Tech. Math. Sci. Mathematics 41 (2021), no. 4, 1-12.

[26] T.S. Kopaliani, Infimal convolution and Muckenhoupt $A_{p(\cdot)}$ condition in variable $L^{p}$ spaces, Arch. Math. 89 (2007), no. 2, 185-192.

[27] O. Kovacik, J. Rakosnik, On spaces $L^{p(x)}$ and $W^{k, p(x)}$, Czechoslovak Math. J. 41 (1991), 592-618.

[28] A.K. Lerner, S. Ombrosi, C. Pérez, R.H. Torres, R. Trujillo-González, New maximal functions and multiple weights for the multilinear Calderón-Zygmund theory, Adv. Math. 220 (2009), 1222-1264.

[29] Y. Lin, Strongly singular Calderón-Zygmund operator and commutator on Morrey type spaces, Acta Math. Sin. (Engl. Ser.) 23 (2007), no. 11, 2097-2110.

[30] Y. Lin, Sh. Lu, Strongly singular Calderón-Zygmund operators and their commutators, Jordan Journal of Mathematics and Statistics 1 (2008), no. 1, 31-49.

[31] Z.G. Liu, S.Z. Lu, Endpoint estimates for commutators of Calderón-Zygmund type operators, Kodai Math. J. 25 (2002), no. 1, 79-88.

[32] G. Lu, P. Zhang, Multilinear Calderón-Zygmund operators with kernels of Dini's type and applications, Nonlinear Anal. 107 (2014), 92-117.

[33] T. Mizuhara, Boundedness of some classical operators on generalized Morrey spaces, Harmonic Analysis (Sendai, 1990), S. Igari, Ed, ICM 90 Satellite Conference Proceedings, Springer, Tokyo, Japan (1991), 183-189.

[34] Y. Mizuta, T. Shimomura, Sobolev embeddings for Riesz potentials of functions in Morrey spaces of variable exponent, J. Math. Soc. Japan 60 (2008), no. 2, 583-602.

[35] C.B. Morrey, On the solutions of quasi-linear elliptic partial differential equations, Trans. Amer. Math. Soc. 43 (1938), 126-166.

[36] E. Nakai, Hardy-Littlewood maximal operator, singular integral operators and Riesz potentials on generalized Morrey spaces, Math. Nachr. 166 (1994), 95-103.

[37] M.M. Rao, Z.D. Ren, Theory of Orlicz spaces, Marcel Dekker Inc., New York (1991).

[38] Y. Sawano, A thought on generalized Morrey spaces, J. Indonesian Math. Soc. 25 (2019), no. 3, 210-281.

[39] E.M. Stein, Harmonic Analysis: Real Variable Methods, Orthogonality and Oscillatory Integrals, vol. 43 of Princeton Mathematical Series, Princeton University Press, Princeton, NJ, USA, 1993.

[40] J. Tan, Z. Liu, J. Zhao, On some multilinear commutators in variable Lebesgue spaces, J. Math. Inequal. 11 (2017), no. 3, 715-734.

[41] H. Wang, Boundedness of $\theta$-type Calderón-Zygmund operators and commutators in the genralized weighted Morrey spaces, J. Funct. Spaces 2016, Art. ID 1309348, 18 pp.

[42] Jingshi $\mathrm{Xu}$, Generalized commutators of multilinear singular integrals, Proc. Razmadze Math. Inst. 142 (2006), 109-122. 
[43] K. Yabuta, Generalizations of Calderón-Zygmund operators, Studia Math. 82 (1985), 17-31.

[44] P. Zhang, J. Sun, Commutators of multilinear Calderón-Zygmund operators with kernels of Dini's type and applications, J. Math. Inequal. 13 (2019), no. 4, 1071-1093.

Vagif S. Guliyev

Institute of Applied Mathematics, Baku State University, Baku, Azerbaijan Dumlupinar University, Department of Mathematics, Kutahya, Turkey

Institute of Mathematics and Mechanics of NAS of Azerbaijan, Baku, Azerbaijan

E-mail address: vagif@guliyev.com

Afag F. Ismayilova

Azerbaijan University of Cooperation, Baku, Azerbaijan

E-mail address: afaismayilova28@gmail.com

Received: May 1, 2021; Revised: July 13, 2021; Accepted: July 24, 2021 